\title{
Initial risk class and length of hospital stay in community-acquired pneumonia
}

\author{
R. Menéndez, D. Ferrando, J.M. Vallés, E. Martínez, M. Perpiñá
}

\begin{abstract}
Initial risk class and length of hospital stay in community-acquired pneumonia. $R$. Menéndez, D. Ferrando, J.M. Vallés, E. Martínez, M. Perpiñá. (C) ERS Journals Ltd 2001.

ABSTRACT: The total medical costs of community-acquired pneumonia are directly related to the costs of hospital admission and length of stay. The aim of the present study was to evaluate the reasons for prolonged duration of stay in patients stratified in five risk classes for death, and to identify factors associated with prolonged stay.

The study population consisted of 295 patients. According to lower (classes I, II, III) or to higher (classes IV, V) risk, the target duration of hospitalization was set at 5 and 7 days, respectively. The causes of prolonged hospitalization were classified as pneumonia-related, complications, unstable comorbid diseases and nonclinical factors.

The overall percentage of patients with appropriate duration of hospitalization was $32 \%$. Causes of prolonged hospitalization were related mainly to pneumonia $(32 \%)$ from all risk classes. Morbid complications and instability of the underlying illness were greater in class $\mathrm{V}$ patients. Nonclinical factors were present in $29.5 \%$ of cases. Hypoxaemia, anaemia, hypoalbuminaemia, and complications appearing before $72 \mathrm{~h}$ were associated with prolonged hospitalization.

The cause of prolonged hospitalization of patients with community-acquired pneumonia is multifactorial, depending mainly on pneumonia and comorbid conditions but there is a large number of unnecessary hospitalization days that could be reduced by improving the efficiency of hospital care.

Eur Respir J 2001; 18: 151-156.
\end{abstract}

Service of Pneumology, Hospital Universitario $\mathrm{La} \mathrm{Fe}$, Valencia, Spain.

\author{
Correspondence: R. Menéndez \\ Servicio de Neumología \\ Hospital Universitario $\mathrm{La} \mathrm{Fe}$ \\ Avda. de Campanar 21 \\ E-46009 Valencia \\ Spain \\ Fax: 34963987397
}

Keywords: Community-acquired pneumonia

length of stay

risk class

Received: October 172000

Accepted after revision March 152001
Community-acquired pneumonia (CAP) has an estimated incidence of $1-5$ per 1,000 of the population, with $\sim 30 \%$ of patients admitted to hospital for treatment. Although most patients are treated on an ambulatory basis, total direct medical costs of pneumonia are dominated by the costs of hospital admission and, therefore, directly related to the length of hospital stay [1-3]. The decision to admit patients to hospital is based on the presence of various risk factors for death or for a complicated course, as suggested by several guidelines and recent studies [4-7]. However, little information exists on the safety and optimal duration of hospitalization.

Generally, patients with bacterial infections stay in hospital for $7-10$ days [8-10].

The length of hospitalization is influenced by underlying comorbid illness, the development of medical complications [11-12] and severity of illness at presentation [7]. For low-risk pneumonia patients, Weingarten et al. [13] proposed a practice guideline that has the potential to significantly and safely reduce the duration of hospital stay.

The purposes of this study were to determine the reasons for prolonged duration of stay for patients hospitalized with pneumonia, stratified in risk classes according to a validated model for death [7], and to identify associated factors based on clinical variables recorded within $72 \mathrm{~h}$ of admission.

\section{Methods}

A prospective study was carried out in a public acute-care teaching hospital in Valencia (Spain) serving a population of 400,000 . Adult patients ( $>16$ yrs of age) were included in the study if they had symptoms compatible with CAP and evidence of a new chest radiographic infiltrate without any other alternative diagnosis during the entire follow-up period (January 1998 to March 1999). Patients with immunosuppression including seropositivity for the human immunodeficiency virus, those who had been hospitalized in the previous 15 days, or those with a suspicion of lung abscess, aspiration, necrotizing pneumonia or tuberculosis were excluded. Patients were hospitalized following recommendations of the Spanish Society of Pneumology and Thoracic Surgery [6], which are very similar to those of the American Thoracic Society (ATS) [4].

A prospective protocol was developed, which was presented to all the medical personnel of the Pneumology service (Hospital Universitario La fe, 
Valencia, Spain), and which comprised: evaluation of initial severity, a pre-established target duration of hospitalization, evaluation of causes of prolonged stay, and a control assessment 30 days after discharge. Patients were assigned to risk classes $\mathrm{I}-\mathrm{V}$ as defined by FINE et al. [7]. According to this classification, for class I - III patients the target duration of hospitalization was set at 5 days, and for class IV $-V$ patients at 7 days. The length of hospital stay was restricted to 5 and 7 days, respectively, as these were the median number of days for class I patients and for the total number of patients in the study of FINE et al. [7].

The attending physician was aware of the patients' risk classification and was responsible for hospital discharge although they did not receive written or oral recommendations for each patient. The physician's decision and potential reasons for prolonged duration of hospitalization (i.e. $>5$ days for class I-III patients and $>7$ days for class $\mathrm{IV}-\mathrm{V}$ patients) were assessed by an independent physician following hospital discharge, who also graded the severity of illness at presentation. An appointment was made for a control visit 30 days after hospital discharge. Readmission and return to normal activities were also recorded.

The severity of illness at presentation was evaluated within $24 \mathrm{~h}$ of admission, following a prediction rule for prognosis developed by FINE et al. [7]. Other data evaluated included any history of alcohol abuse, chronic obstructive pulmonary disease (COPD), diabetes mellitus, previous antibiotic therapy and regular treatment with inhaled steroids, as well as blood cell count, serum albumin and liver enzyme levels, and the number of pulmonary lobes showing an infiltrate on the initial chest radiograph.

Reasons for continued hospitalization were assessed by medical record review after discharge from the hospital. A protocol similar to that described by WeINGARTEn et al. [13] was also used to assess clinical reasons, and evaluation of other nonclinical reasons. Criteria for prolonged hospitalization were categorized in four categories as outlined in table 1 . Total length of hospital stay per patient was calculated by subtracting the admission date from the discharge date.

In addition, radiographical and analytical control data were recorded for patients alive at 30 days when deemed necessary, together with information on complications related to pneumonia and/or underlying disease that required treatment in the outpatient setting, re-admission rate due to pneumonia- and/or comorbid disease-related problems, and days for return to usual activities or to work.

\section{Statistical analysis}

Primary end-points included adherence to guidelines for hospital discharge, duration of hospitalization, and mortality for risk classes of FINE et al. [7]. The Mann-Whitney U-test, Spearman's correlation coefficient, and the Chi-squared test were used to compare parameters associated with prolonged hospitalization. Causes of prolonged hospitalization
Table 1.-Categorization of causes of prolonged hospitalization

Causes

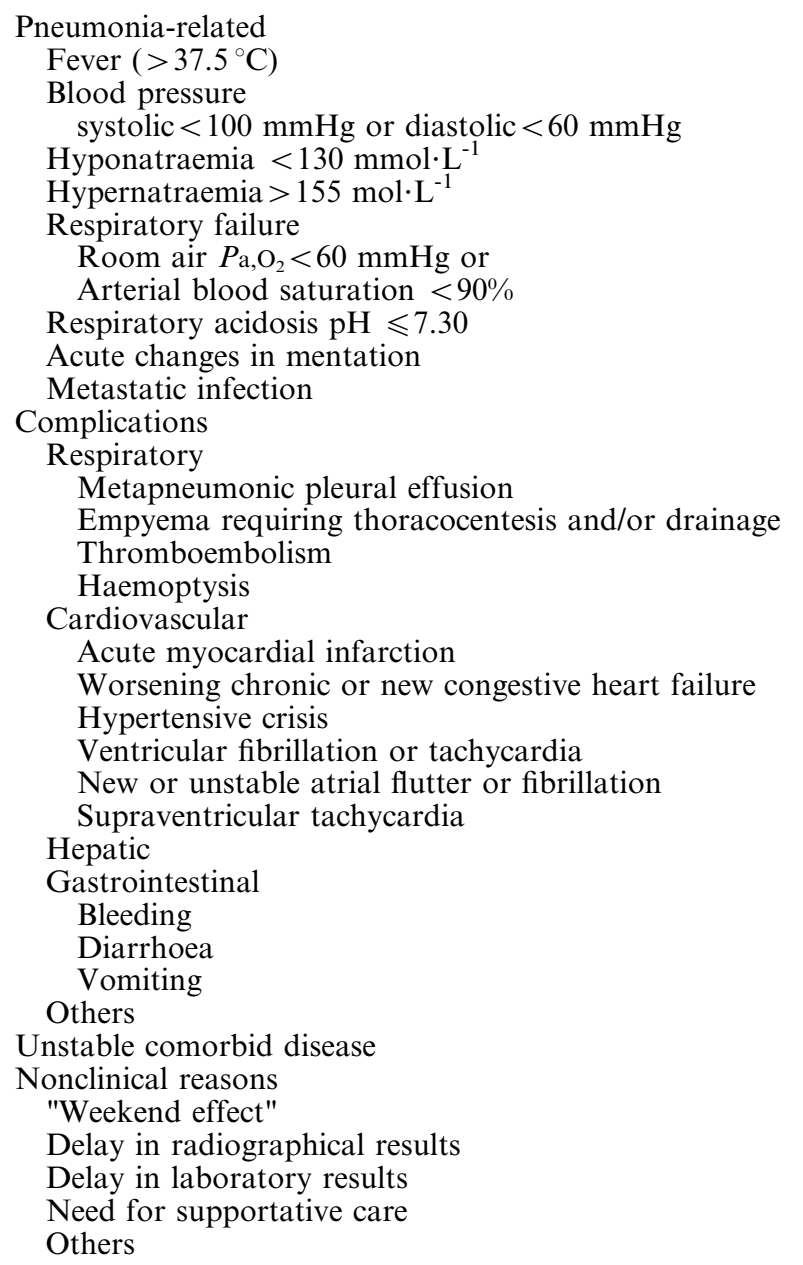

$\mathrm{Pa}, \mathrm{O}_{2}$ : arterial oxygen tension.

depending on initial risk class were analysed by analysis of variance (ANOVA); when statistical significance was achieved a post hoc correction using the Bonferroni test was performed within the five risk classes. The Kruskal-Wallis test was used when variables did not follow a normal distribution. Statistical significance was set at $\mathrm{p}<0.05$.

\section{Results}

A total of 295 inpatients with CAP were included in the study. As shown in table $2,59 \%$ of patients were males and the mean age of the population was $70 \mathrm{yrs}$. Congestive heart failure, COPD, diabetes, and cerebrovascular disease were the most frequent co-existing conditions. Reasons for hospitalization in the 64 patients with risk class I and II included in Sociedad Española de Neumología y Cirugía Torácica (SEPAR) guidelines were: comorbidity, 17 (two COPD and one asthma in risk class I and four COPD, four cardiovascular, three neoplasic, two hepatic and one 
Table 2. - Demographic and clinical characteristics of 295 patients with community-acquired pneumonia admitted to hospital

\begin{tabular}{lcr}
\hline Data & Number & Per cent \\
\hline Age, yrs & $70 \pm 15$ & \\
Sex, M/F & $175: 120$ & \\
Co-existing conditions & & \\
Congestive heart failure & 98 & 33.2 \\
COPD & 75 & 25.4 \\
Diabetes mellitus & 61 & 20.7 \\
Cerebrovascular disease & 32 & 10.8 \\
Renal disease & 18 & 6.1 \\
Neoplastic disease & 14 & 4.7 \\
Liver disease & 11 & 3.7 \\
Risk class for death & & \\
I & 23 & 7.8 \\
II & 41 & 13.9 \\
III & 61 & 20.7 \\
IV & 121 & 41.0 \\
V Length of stay days & 49 & 16.6 \\
Deaths & $9.6 \pm 5$ & \\
\hline
\end{tabular}

Data are presented as mean \pm SD or subject numbers. M: male; F: female; COPD: chronic obstructive pulmonary disease; I, II, III: low risk; IV, V: high risk.

cerebrovascular diseases in risk class II); age $>65 \mathrm{yrs}$, 11; prior treatment failure, nine; pleural effusion, 10; multilobar pneumonia, eight; respiratory failure, five; respiratory rate $(>30)$, two; increased urea, one; and leukocytosis $(>30,000)$, one. Antibiotic therapy included the administration of $\beta$-lactam agents in 83 patients, quinolones or macrolides in 27 , and a combination of $\beta$-lactams or quinolones and macrolides in 185. Adherence to SEPAR guidelines with respect to empirical treatment was achieved in $65.8 \%$.
Prior ambulatory antimicrobial treatment had been prescribed in $36 \%$. The median duration of hospitalization was 9 days.

Thirty-one patients died giving a mortality rate of $10.5 \%$. The distribution of patients according to risk class for death is shown in table 2. More than half of the patients were in the highest risk classes. The group of patients who died had a mean age of $77.6 \mathrm{yrs}$ and co-existing conditions were present in $26(84 \%)$. Mean \pm SD length of stay in hospital was $9.3 \pm 7.4$ days. Twenty-seven $(87.1 \%)$ of the 31 patients who died had been assigned to the highest risk class (IV V). Causes of death were acute respiratory failure in eight patients, heart failure in eight, septic shock in four, multi-organ failure in three, decompensation of the comorbid illness in four, and progression of pneumonia in four.

The overall percentage of patients who stayed longer than expected was $68 \%$. The causes of prolonged hospitalization in surviving patients according to risk classes are shown in table 3. A significantly higher rate of prolonged hospitalization was found in risk class III $(86.9 \%)$ compared to risk class IV $(58.7 \%) \quad(\mathrm{p}<0.05)$. Causes of prolonged hospitalization were associated with pneumonia in $32 \%$ of cases, mostly due to respiratory failure; medical complications occured in $14 \%$ and unstability of the comorbid illness in $12 \%$ (table 3). Statistical differences were found among the five risk classes for pneumonia-related reasons, which were higher in risk class III compared to risk class IV (Bonferroni test, $\mathrm{p}<0.05)$. Complications were especially frequent among patients in risk class $\mathrm{V}$ and strikingly frequent in risk class I; the differences were almost statistically significant $(\mathrm{p}=0.06)$.

Seventy per cent of complications were already

Table 3. - Causes of continued hospitalization according to risk classes

\begin{tabular}{|c|c|c|c|c|c|c|c|}
\hline \multirow[b]{2}{*}{ Data } & \multicolumn{7}{|c|}{ Risk class $^{+}$} \\
\hline & Total $\mathrm{n}$ & I & II & I & IV & V & $\mathrm{p}$-value \\
\hline Patients $n$ & 295 & 23 & 41 & 61 & 121 & 49 & 0.01 \\
\hline Length of stay days ${ }^{+}$ & & 7 & 8 & 9 & 9 & 10 & 0.01 \\
\hline Deaths & $31(10.5)^{+}$ & $1(43)$ & & $3(4.9)$ & $12(9.9)$ & $15(30.6)$ & $<0.001$ \\
\hline Continued hospitalisation & $201(76.1)^{+}$ & $16(69.5)$ & $28(68.3)$ & $53(86.9)$ & $71(58.7)$ & $33(67.3)$ & 0.008 \\
\hline Pneumonia-related reasons & $85(32.2)$ & $4(18.2)$ & $12(29.3)$ & $28(48.3)$ & $29(26.6)$ & $12(35.3)$ & 0.04 \\
\hline Fever & $28(10.6)$ & $4(18.2)$ & $4(9.8)$ & $11(18.9)$ & $7(6.4)$ & $2(5.9)$ & \\
\hline Respiratory failure & $47(17.8)$ & & $6(14.6)$ & $14(24.1)$ & $18(16.5)$ & $9(26.5)$ & \\
\hline Metastatic infection site & $5(1.9)$ & $1(4.5)$ & $3(7.3)$ & & & $1(2.9)$ & \\
\hline Acute changes in mentation & $4(1.5)$ & & $1(2.4)$ & & $2(1.8)$ & $1(2.9)$ & \\
\hline Hyponatraemia, hypernatraemia & $3(1.1)$ & $1(4.5)$ & & $1(1.7)$ & $1(0.9)$ & & \\
\hline Other* & $3(1.1)$ & & & $1(1.7)$ & & $2(5.9)$ & \\
\hline Complications & $36(13.6)$ & $5(22.7)$ & $3(7.3)$ & $6(10.3)$ & 13 (11.9) & $9(26.5)$ & 0.06 \\
\hline Unstable underlying illness & $32(12.1)$ & $2(9.1)$ & $3(7.3)$ & $7(12.1)$ & $13(11.9)$ & $9(26.5)$ & NS \\
\hline Nonclinical factors & $78(29.5)$ & $5(22.7)$ & $16(39.0)$ & $20(34.5)$ & $28(25.7)$ & $9(26.5)$ & NS \\
\hline "Weekend effect" & $24(9.1)$ & $2(9.1)$ & $2(4.9)$ & $10(17.2)$ & $8(7.3)$ & $2(5.9)$ & \\
\hline Delay in radiographic results & $20(7.5)$ & $1(4.5)$ & $4(9.7)$ & $5(8.6)$ & $9(8.3)$ & $1(2.9)$ & \\
\hline Delay in laboratory results & $21(7.9)$ & $2(9.1)$ & $7(17.1)$ & $2(3.4)$ & $8(7.3)$ & $2(5.9)$ & \\
\hline Need of supportive care & $7(2.6)$ & & $1(2.4)$ & $1(1.7)$ & $2(1.8)$ & $3(8.8)$ & \\
\hline Other & $6(2.3)$ & & $2(4.9)$ & $2(3.4)$ & $1(0.9)$ & $1(2.9)$ & \\
\hline
\end{tabular}

Data are expressed in absolute number (\%).; Some patients may have had more than one cause for continued hospitalization. I, II, III: low risk; IV, V: high risk; NS: nonsignificant. ${ }^{+}$: including the whole sample group; *: severe diabetic decompensation, haemolytic anemia, electrolyte abnormalities other than hypo- or hypernatraemia. 
Table 4.- Reasons for medical care after discharge

\begin{tabular}{|c|c|c|c|c|c|c|}
\hline \multirow{2}{*}{ Reason } & \multirow{2}{*}{ Patients $n$} & \multicolumn{5}{|c|}{ Risk class } \\
\hline & & I & II & III & IV & $\mathrm{V}$ \\
\hline Persistence of symptoms & 14 & 2 & 2 & 3 & 6 & 1 \\
\hline $\begin{array}{l}\text { New symptoms or fever } \\
\text { after an asymptomatic } \\
\text { period }\end{array}$ & 11 & 2 & & 1 & 7 & 1 \\
\hline $\begin{array}{l}\text { Decompensation of } \\
\text { co-existing condition } \\
\text { or mild complications }\end{array}$ & 6 & & & & 6 & \\
\hline Re-admission & 9 & & & 3 & 4 & 2 \\
\hline
\end{tabular}

present on admission or developed within $24 \mathrm{~h}$, while $90 \%$ of complications appeared within $72 \mathrm{~h}$ of hospital admission. Nonclinical factors, in particular the "weekend effect" and delays in availability of radiographic or laboratory results, accounted for prolonged stay in $29.5 \%$ of cases, especially in the three lowest risk classes, although the differances were not significant.

The reasons for remaining hospitalized after the target duration, if patients who died later are included, remained almost the same: pneumonia-related reasons $30.8 \%$, complications $13.9 \%$, unstable underlying disease $13.6 \%$ and nonclinical factors $29.5 \%$.

Thirty-one patients received medical care (table 4 ) in the outpatient setting due to persistence or worsening of cough, dyspnoea, or expectoration, new symptoms or fever after an asymptomatic period, and decompensation of co-existing condition or mild complications. However, only nine patients were re-admitted (hospital re-admission rate of 3.4\%). All re-admissions occurred in patients with prolonged hospitalization. Patients were able to return to normal activities after an average of 24 days. There were no significant differences in the average time to return to daily activities between patients with appropriate and prolonged length of stay, or among patients in the different risk classes.

Prolonged hospitalization was significantly associated with neoplastic disease, anaemia, and development of respiratory, cardiovascular, renal, and gastrointestinal complications within $72 \mathrm{~h}$ of hospital admission. Serum albumin correlated inversely with prolonged hospitalization $(\mathrm{r}=-0.18, \mathrm{p}<0.05)$. Initial oxygen tension in arterial blood $\left(\mathrm{Pa}_{\mathrm{a}} \mathrm{O}_{2}\right)$ also showed an inverse correlation with prolonged hospitalization $(\mathrm{r}=-0.29, \mathrm{p}<0.05)$. The proportion of patients treated according to SEPAR guidelines, and who adjusted to protocol length of stay was $34 \%$ versus $26 \%$ in the rest $(p>0.05)$. Seventy-one per cent of patients who received prior ambulatory antimicrobial treatment stayed longer than the target duration versus $67 \%$ of those who did not receive it $(\mathrm{p}>0.05)$.

\section{Discussion}

It was found that pneumonia-related reasons were responsible for prolonged hospitalization in $32 \%$ of patients, and that time to reach stability of a coexisting condition and/or morbid complications were responsible in more than one-third of patients in the highest risk class. Moreover, potentially unnecessary hospitalization days were found in $29 \%$ of patients due the "weekend effect" and/or delay in the availability of chest radiographs or results of laboratory tests. Clinical factors associated with continued hospitalization included initial hypoxaemia, anaemia, neoplastic disease and complications within $72 \mathrm{~h}$ after admission.

In the present study, like others, the criteria for admission were in accordance with recommendations of the ATS [4] and SEPAR. These criteria include specific risk factors not only for a mortality, but also for a complicated course, such as comorbidity or age $>65 \mathrm{yrs}$, which were in fact found in some patients in risk classes I and II. Moreover, as recognized by FINE et al. [7], the prediction rule has potential limitations: it does not estimate important clinical features, such as multilobar pneumonia or prior treatment failure, and may minimize the significance of notable signs, such as those of respiratory failure or severe comorbidity, especially in young patients, in whom the low age would not sum up to the 71 points required to reach class III.

A total of $32 \%$ of patients were discharged from the hospital on the expected date or before, whereas prolonged hospitalization was recorded in the other $68 \%$ of cases. In the present study, target days 5 and 7 (median of class I and of the whole group of FINE et al. [7]) were chosen instead of a more conservative length of stay of 7 and 9 days (median of class III and $\mathrm{V}$, respectively) because the aim was to study causes of prolonged stays and it has been reported as a frequent event that patients remain hospitalized for about two days after reaching stability [14]. In fact, in $\leqslant 50 \%$ of patients in the study of HALM et al. [15], the median time to achieve overall clinical stability was 4-6 days for risk classes I- III and 6-7 days for risk classes IV and V. As shown by others [15-17], pneumoniarelated problems were the most frequent cause of continued hospitalization in all risk classes. In the present study, class III patients showed the highest percentage of pneumonia-related causes responsible for prolonged hospitalization, so that only $50 \%$ of these patients reached clinical stability in 5 days. If the target time to be discharged in class III patients had been 7 days, $40 \%$ of hospital stays would have been considered appropriate. MCCORMICK et al. [18] showed that interhospital variation in the lengths of stay was particularly substantial in the moderate-risk category (risk class III).

Instability of comorbid disease was the most common reason for continued hospitalization in the high-risk category. FINE and coworkers [14, 19] identified treatment of comorbid illness as one of the factors recognized by physicians to cause prolonged hospital stay in CAP. However, continued hospital stay may be justified for the adjustment of medication for cardiac disease or diabetes mellitus, as explicitly mentioned in the appropriateness evaluation protocol (AEP) for justifying a hospital day for pneumonia patients [20]. A target value for length of stay is difficult to establish when care of comorbid conditions is considered. According to the AEP [20], a decision 
for hospital discharge can be appropriate when adjustment of medications within the past $48 \mathrm{~h}$ has not been required.

It has been shown that wide discretion in physicians discharge decisions correlate with differences in patient outcome [21, 22]. In fact, no re-admissions occurred among the patients with appropriate hospital stay, so that in the absence of problems, the fixed targets of 5 and 7 days depending on the severity of illness are safe. WEINGARTEN and coworkers [13, 23, 24] investigated the safety and effectiveness of a practice guideline that provided information about switching patients from parenteral to oral antimicrobials and early hospital discharge. They found a high compliance and that patient outcomes remained unchanged $[13,23,24]$. In the multicentre study of McCormick et al. [18], medical outcomes including mortality, hospital re-admission rate, and dates of return to usual activities and to work were similar in patients admitted to hospital with differences in the length of stay. Likewise, in the present study, return to usual activities was neither associated with inadequacy to target length of stay, nor with risk classes for death.

Interestingly, nonclinical factors, especially delays in having available results of radiographic or laboratory tests and the "weekend effect", accounted for failure of the fixed criteria in $29.5 \%$ of cases, mainly among patients in the three lowest risk categories. Accordingly, improving the efficiency of hospital care should reduce the mean duration of hospitalization for this disease without adversely affecting outcome. Additional measures may also be helpful, such as assessment of the patient's status before the control visit at 30 days and improving the quality of discharge planning [25]. Although short lengths of stay may be associated with cost-shifting (reductions in costs in the hospital off-set by increases in costs after hospital discharge) [26], most patients who are at low risk for mortality from CAP prefer outpatient treatment [27].

Morbid complications, particularly respiratory and cardiac complications were the third largest cause of hospital stay longer than expected. These findings are consistent with the prospective study of FINE et al. [12], who reported that most surviving inpatients $(69 \%)$ and almost all who died $(94.4 \%)$ had one or more medical complications, with respiratory failure and congestive heart failure the most prevalent. It should be noted that in the present study, almost $90 \%$ of medical complications occurred within the first $72 \mathrm{~h}$ of admission. This is a clinically relevant finding since the likelihood of severe complications after 3 days is substantially reduced.

Several initial factors were associated with prolonged stay, such as hypoxaemia, anaemia, neoplastic disease, level of albumin, and complications appearing during the first $72 \mathrm{~h}$. Hypoxaemia is a standard criterion for admission to hospital and supports consideration for intensive care [24]. In the present study, the degree of hypoxaemia correlated with prolonged length of stay. HALm et al. [15] found that the median time to stabilization was longer to achieve oxygen saturation $(\geqslant 90 \%)$ than for other vital signs.

In conclusion, multiple causes were associated with prolonged hospitalization in patients with communityacquired pneumonia: the most important were pneumonia-related clinical causes and nonclinical factors, and less frequently complications and instability of underlying conditions. Clinical factors associated with prolonged length of stay were initial hypoxaemia, anaemia, neoplastic disease and complications arising within $72 \mathrm{~h}$ after admission. Nonclinical factors generated a large number of unnecessary hospitalization days, even among patients in the lowest risk classes, which should be reduced by improving the efficiency of hospital care and the quality of the postdischarge treatment plan.

Acknowledgements. The investigators thank M. Pulido for editing the manuscript and editorial assistance.

\section{References}

1. Niederman MS, McCombs JS, Unger AN, Kumar A, Popovian R. The cost of treating community-acquired pneumonia. Clin Ther 1998; 20: 820-837.

2. Guest JF, Morris A. Community-acquired pneumonia: the annual cost to the National Health Service in the UK. Eur Respir J 1997; 10: 1530-1534.

3. Lave JR, Fine MJ, Sankey SS, Hanusa BH, Weissfeld LA, Kapoor WN. Hospitalized pneumonia: outcomes, treatment patterns, and costs in urban and rural areas. J Gen Intern Med 1996; 11: 415-421.

4. American Thoracic Society. Guidelines for the initial management of adults with community-acquired pneumonia: diagnosis, assessment of severity, and initial antimicrobial therapy. Am Rev Respir Dis 1993; 148: $1418-1426$.

5. Bartlett JG, Breinman RF, Mandell LA, File TM. Community-acquired pneumonia in adults: guidelines for management. Clin Infect Dis 1998; 26: 811-838.

6. Dorca J, Bello S, Blanquer J, et al. Diagnóstico y tratamiento de la neumonía adquirida en la comunidad. Arch Bronconeumol 1997; 33: 240-246.

7. Fine MJ, Auble TE, Yealy DM, et al. A prediction rule to identify low-risk patients with communityacquired pneumonia. N Engl J Med 1997; 336: $243-$ 250.

8. National Center for Health Statistics: detailed diagnoses and procedures, national hospital discharge survey, 1990. No. 113, Series 13. Washington, OL, NCHS, 1992.

9. Marras TK, Chan CK. Use of guidelines in treating community-acquired pneumonia. Chest 1998; 113: $1689-1694$.

10. Riquelme RA, Torres A, El-Ebiary M, et al. Community-acquired pneumonia in the elderly. A multivariate analysis of risk and prognostic factors. $\mathrm{Am}$ J Respir Crit Care Med 1996; 154: 1450-1455.

11. Fine MJ, Smith MA, Carson CA, et al. Prognosis and outcomes of patients with community-acquired pneumonia: a meta-analysis. JAMA 1996; 275: 134-141.

12. Fine MJ, Stone RA, Singer DE, et al. Processes and outcomes of care for patients with communityacquired pneumonia. Results from the Pneumonia Patient Outcomes Research Team (PORT) Cohort Study. Arch Intern Med 1999; 159: 970-980. 
13. Weingarten SR, Riedinger MS, Hobson $\mathrm{P}$, et al. Evaluation of a pneumonia practice guideline in an intervention trial. Am J Respir Crit Care Med 1996; 153: $1110-1115$.

14. Fine MJ, Medsger AR, Stone RA, et al. The hospital discharge decision for patients with communityacquired pneumonia. Results from the Pneumonia Patient Outcomes Research Team Cohort Study. Arch Intern Med 1997; 157: 47-56.

15. Halm EA, Fine MJ, Marrie TJ, et al. Time to clinical stability in patients hospitalized with communityacquired pneumonia. Implications for practice guidelines. JAMA. 1998; 279: $1452-1457$.

16. Daikufu R, Movahhed H, Fotheringham N, Bear MB, Nelson S. Time to resolution of morbidity: an endpoint for assessing the clinical cure of community-acquired pneumonia. Respir Med 1996; 90: $587-$ 592.

17. Metlay JP, Atlas SJ, Borowsky LH, Singer DE. Time course of symptom resolution in patients with community-acquired pneumonia. Respir Med 1998; 92: $1137-1142$.

18. McCormick D, Fine MJ, Coley CM, et al. Variation in length of hospital stay in patients with communityacquired pneumonia: are shorter stays associated with worse medical outcomes? Am J Med 1999; 107: 5-12.

19. Fine JM. Pneumonia in the elderly: the hospital admission and discharge decision. Semin Respir Infect 1990; 5: 303-313.

20. Hartz AJ, Bade PF, Sigmann P, Guse C, Epple P, Goldberg KC. The evaluation of screening methods to identify medically unnecessary hospital stay for patients with pneumonia. Int $J Q$ Health Care 1996; 8: $3-11$.

21. Hand R, Walden LK, Inczauskis D. Mortality and length of stay as performance indicators for pneumonia in the elderly. J Invest Med 1997; 45: 183-190.

22. Fine MJ, Singer DE, Phelps AL, Hanusa BH, Kapoor WN. Differences in length of hospital stay in patients with community-acquired pneumonia: a prospective four-hospital study. Med Care 1993; 31: 371-380.

23. Rhew DC, Hackner D, Henderson L, Ellrodt AG, Weingarten SR. The clinical benefit of in-hospital observation in 'low-risk' pneumonia patients after conversion from parenteral to oral antimicrobial therapy. Chest 1998; 113: 142-146.

24. Weingarten SR, Riedinger MS, Varis G, et al. Identification of low-risk hospitalized patients with pneumonia. Implications for early conversion to oral antimicrobial therapy. Chest 1994; 105: 1109-1115.

25. Calkins DR, Davis RB, Reiley $\mathrm{P}$, et al. Patientphysician communication at hospital discharge and patients' understanding of the postdischarge treatment plan. Arch Intern Med 1997; 157: 1026-1030.

26. Weingarten S, Riedinger MS, Sandhu M, et al. Can practice guidelines safely reduced hospital length of stay? Results from a multicenter interventional study. Am J Med 1998; 105: 33-40.

27. Coley CM, Li Y-H, Medsger AR, et al. Preferences for home vs hospital care among low-risk patients with community-acquired pneumonia. Arch Intern Med 1996; 156: 1565 - 1571 . 